\title{
Short-Term Symptomatic Relief in Gastroesophageal Reflux Disease: A Comparative Study of Esomeprazole and Vonoprazan
}

\author{
Kouichi Sakurai ${ }^{1} \cdot$ Hiroko Suda $^{1} \cdot$ Satomi Fujie ${ }^{1}$ 'Takayuki Takeichi ${ }^{1} \cdot$ Ayako Okuda $^{1} \cdot$ Tetsuya Murao $^{1}$. \\ Kiwamu Hasuda ${ }^{1} \cdot$ Masahiro Hirano $^{2} \cdot$ Kiyoharu Ito $^{3} \cdot$ Katsuie Tsuruta $^{4} \cdot$ Masahiro Hattori $^{1}$
}

Received: 24 July 2018 / Accepted: 1 November 2018 / Published online: 10 November 2018

(c) The Author(s) 2018

\begin{abstract}
Background and Aim Proton pump inhibitors and vonoprazan (a potassium-competitive acid blocker) are recommended as first-line treatments for gastroesophageal reflux disease (GERD). However, few reports have investigated the onset of action of these agents for GERD symptom relief. The present study compared the symptom relief of esomeprazole with that of vonoprazan via monitoring self-reported GERD symptoms after treatment initiation.

Methods This was a prospective, multicenter, randomized, open-label, parallel group, comparative clinical study between esomeprazole (20 mg/day) and vonoprazan $(20 \mathrm{mg} /$ day) administered for 4 weeks to patients with GERD symptoms. Patients who had scores $\geq 8$ on the Gastroesophageal Reflux Disease Questionnaire (GerdQ) were defined as having GERD and enrolled in this study. Sixty patients were randomly assigned to either the esomeprazole group $(n=30)$ or the vonoprazan group $(n=30)$. Treatment response rates in each drug group were evaluated according to the GerdQ. The Frequency Scale for the Symptoms of GERD (FSSG) scores from the 1st day after treatment initiation and the Global Overall Symptom (GOS) scale scores during treatment were also evaluated.

Results At 4 weeks, the treatment response rates for symptom relief according to the GerdQ were $88.0 \%$ in the esomeprazole group and $81.8 \%$ in the vonoprazan group. The GOS scales, which reflect the impact of GERD symptoms, were similar for both groups. The FSSG scores decreased from the 1st to the 14th day in both groups.

Conclusions There were no substantial differences in the symptom relief between the two groups at any time point in this short-term study.
\end{abstract}

Keywords Esomeprazole $\cdot$ Vonoprazan $\cdot$ Symptom relief $\cdot$ Gastroesophageal reflux disease

Kouichi Sakurai

sakuraiko@jcom.zaq.ne.jp

Hiroko Suda

sudahiro0825@gmail.com

Satomi Fujie

sa-fu@ hattori-clinic.com

Takayuki Takeichi

ta-ta@hattori-clinic.com

Ayako Okuda

a-oku@hattori-clinic.com

Tetsuya Murao

te-mura@hattori-clinic.com

Kiwamu Hasuda

ki-hasu@hattori-clinic.com

Masahiro Hirano

gihirano@gaea.ocn.ne.jp
Kiyoharu Ito

itoh@ozakiiin.or.jp

Katsuie Tsuruta

tsurukatsu@gmail.com

Masahiro Hattori

m-hattori@hattori-clinic.com

1 Hattori Clinic, 2-12-35 Shin-machi, Chuo-ku, Kumamoto City, Kumamoto 860-0004, Japan

2 Hirano Gastroenterology Clinic, 2-3029-2, Oonuki-machi, Nobeoka City, Miyazaki 882-0803, Japan

3 Ozaki Clinic, 1-8 Hon-machi, Uto City, Kumamoto 869-0431, Japan

4 Tsuruta Hospital, 10-112 Hotakubohon-machi, Higashi-ku, Kumamoto City, Kumamoto 862-0925, Japan 


\section{Introduction}

The incidence of gastroesophageal reflux disease (GERD) has increased in recent years. In the Japanese society, this increase is due to a decrease in the Helicobacter pylori (H. pylori) infection rate caused by increasing the eradication therapy for atrophic gastritis, the westernization of food habits, obesity, and an aging population with gibbus [1].

GERD is diagnosed via endoscopy and/or clinical symptoms, such as heartburn and acid regurgitation. GERD is classified into two categories: nonerosive reflux disease (NERD) and erosive esophagitis. NERD is characterized by the presence of the typical symptoms of GERD without visible erosive esophagitis on endoscopy. Erosive esophagitis is characterized by injury to the esophageal mucosa on endoscopy $[2,3]$.

The major symptoms of GERD, including heartburn and acid regurgitation, decrease the patient's quality of life (QOL) $[4,5]$. Therefore, improvement in the QOL of these patients by providing rapid relief of symptoms is the primary objective of treatment.

Proton pump inhibitors (PPIs) are the most effective agents for reducing acid secretion, and these agents are recommended as the first-line treatment for GERD [6].

Esomeprazole is known to be as effective as other PPIs for acid suppression [7], and its metabolism is less susceptible to cytochrome P450 2C19 (CYP2C19) polymorphisms [8, 9].

Vonoprazan is a novel potassium-competitive acid blocker (P-CAB) that is known as a new class of gastric acid-suppressing agents. $\mathrm{P}-\mathrm{CAB}$ s block $\mathrm{H}^{+}, \mathrm{K}^{+}$ATPase in a competitive and reversible manner, which results in stronger and more sustained acid suppression than with PPIs [10-13]. The healing rate of reflux esophagitis was not inferior to a PPI (lansoprazole) in a clinical trial, and a possible superior effect was observed in more severe cases [14, 15].

P-CABs exhibit a more rapid onset of action than PPIs and achieve a maximum acid inhibitory effect on the 1st day after treatment, while PPIs require 3-5 days.

However, few studies have examined whether the rapid onset of vonoprazan contributes to the clinical effects on the typical GERD symptoms of heartburn and acid regurgitation.

The aim of this study was to compare the efficacy of $20 \mathrm{mg}$ of esomeprazole and $20 \mathrm{mg}$ of vonoprazan as first-line treatments for the relief of typical GERD symptoms.

\section{Methods}

\section{Patients}

Patients with total scores $\geq 8$ on the Japanese version of the Gastroesophageal Reflux Disease Questionnaire (GerdQ) $[16,17]$ and scores $\geq 2$ for heartburn and/or regurgitation, indicating that they experienced heartburn and/or regurgitation for two or more days per week, were eligible for this study. The exclusion criteria were as follows: less than 20 years of age; past history of total/subtotal gastrectomy, esophageal stenosis, esophageal achalasia, gastrointestinal disease (gastric or duodenal ulcer), acute gastritis, Zollinger-Ellison syndrome, inflammatory bowel disease, or other serious disease (hepatic, renal, respiratory, or cardiac disease); history of malignancy; pregnancy; currently using histamine- 2 receptor antagonists, antacids, gastrointestinal motility stimulants or steroids; and a history of drug allergy to PPIs.

The following medications were not permitted during the study period: nonsteroidal anti-inflammatory drugs (NSAIDs), low-dose aspirin, and antidepressants. PPIs and vonoprazan were discontinued at least 8 weeks prior to study entry.

\section{Study Design and Methods}

This was a prospective, multicenter, randomized, open-label, parallel group, comparative clinical study. Four centers in Japan participated, including the Hattori Clinic, Hirano Gastroenterology Clinic, Ozaki Clinic and Tsuruta Hospital.

The study was conducted in accordance with the Declaration of Helsinki between May 2016 and November 2017 after obtaining approval from the Institutional Review Board of the Hattori Clinic (UMIN000021753). All patients provided written informed consent prior to study enrollment.

Patients were randomly allocated to receive $20 \mathrm{mg}$ of esomeprazole or $20 \mathrm{mg}$ of vonoprazan. We made random allocation lists of the study drugs in a 1:1 ratio and delivered one to each institution. The responsible investigator at each institution managed the list and allocated each study drug according to the list to patients who fulfilled the inclusion and exclusion criteria.

Both drugs were administered orally once daily after dinner for 4 weeks.

GERD symptoms were assessed using the GerdQ, the Frequency Scale for the Symptoms of GERD (FSSG) [18], and the Global Overall Symptom (GOS) scale [19].

The GerdQ is a useful tool for primary care physicians for the diagnosis and management of GERD. It is a selfadministered questionnaire consisting of six questions (1: heartburn, 2: regurgitation, 3: epigastric pain, 4: nausea, 5: sleep disturbance due to heartburn or regurgitation, and 6: use of over-the-counter (OTC) medication to manage heartburn or regurgitation) that is used for symptom-based diagnosis [16, 17]. Questions 1, 2, 5, and 6 are positive predictors of GERD, and questions 3 and 4 are negative predictors. Scores ranging from 0 to 3 indicate symptom frequency per week of positive predictors $(0=$ no symptoms, $1=1$ day, 
$2=2-3$ days, and $3=4-7$ days). The scores are used in reverse for negative predictors.

The FSSG questionnaire comprises twelve questions in two domains, the reflux symptom domain and dysmotility symptom domain. The FSSG uses a 5-point scale (never $=0$, occasionally $=1$, sometimes $=2$, often $=3$, and always $=4$ ).

The GOS scale has been validated for the assessment of upper gastrointestinal symptoms, and it is used to assess GERD symptoms, i.e., heartburn and acid regurgitation. It is also a self-reported questionnaire that assesses the impact of symptoms on a patient's daily life by measuring the severity of eight symptoms (epigastric pain, heartburn, acid regurgitation, epigastric discomfort, nausea, burping, early satiety, and postprandial fullness) on a 7-point Likert scale ranging from 1 (no problem) to 7 (very severe problem).

Each questionnaire was completed by patients at the start of the study, after 1 day, 3 days, 1 week, and 2 weeks, and in the clinic at the 4-week visit. Adverse events (AEs) were defined as undesirable medical symptoms or conditions that occurred after the beginning of treatment, which were collected via investigators' interviews of patients during their 4-week visit or when they visited for their AE.

We performed upper gastrointestinal endoscopy at the start of the study. The diagnosis of erosive esophagitis was made according to the modified Los Angeles (LA) classification (LA grades N, M, A-D) [20, 21]. The Kimura-Takemoto classification was used to classify atrophic gastritis [22]. The study design is shown in Fig. 1.

\section{Evaluation and Statistical Analysis}

The primary endpoint was sufficient relief at 4 weeks as assessed by the GerdQ. Sufficient relief was defined by scores $\leq 1$ for questions $1,2,5$, and 6 . Complete symptom resolution was also assessed by a score of 0 . Scores were assessed at 1,2, and 4 weeks.

FSSG scores were assessed on the 1st, 3rd, 7th, 14th, and 28th day after treatment initiation.
GOS scores were assessed at 2 and 4 weeks, and the number of patients with no or minimum impact on their daily life was calculated from these scores. Sufficient relief was defined by a score $\leq 2$ for each symptom of reflux or regurgitation after treatment, and complete resolution was defined by a score of 1 for each respective symptom.

The secondary endpoints were factors associated with symptom relief.

AEs were recorded throughout the study period.

The primary endpoint was the number of patients who achieved symptom relief on the GerdQ in both treatment regimens, and these values were compared using the Chisquared test. Patients who were lost during follow-up or who did not comply with the protocol were excluded from the analysis. $P$ values $<0.05$ were defined as statistically significant differences. Factors associated with symptom relief after treatment were assessed using logistic regression analysis. Patient characteristics for both regimens and symptom scores on patient questionnaires were analyzed using a parametric or nonparametric model, according to the characteristics of the data. No adjustments were made for multiple comparisons.

\section{Results}

A total of 60 patients provided informed consent and were randomly assigned to the esomeprazole group $(n=30)$ or the vonoprazan group $(n=30)$. Forty-seven patients completed the study ( $n=25$ in the esomeprazole group and $n=22$ in the vonoprazan group). Four patients in the esomeprazole group and 7 patients in the vonoprazan group discontinued treatment by themselves due to improvement in their symptoms, and 1 patient in the esomeprazole group and 1 patient in the vonoprazan group did not visit the clinic according to the defined schedule; these patients were excluded from the efficacy analysis. These exclusions caused an imbalance in the number of patients allocated to each drug; however, it

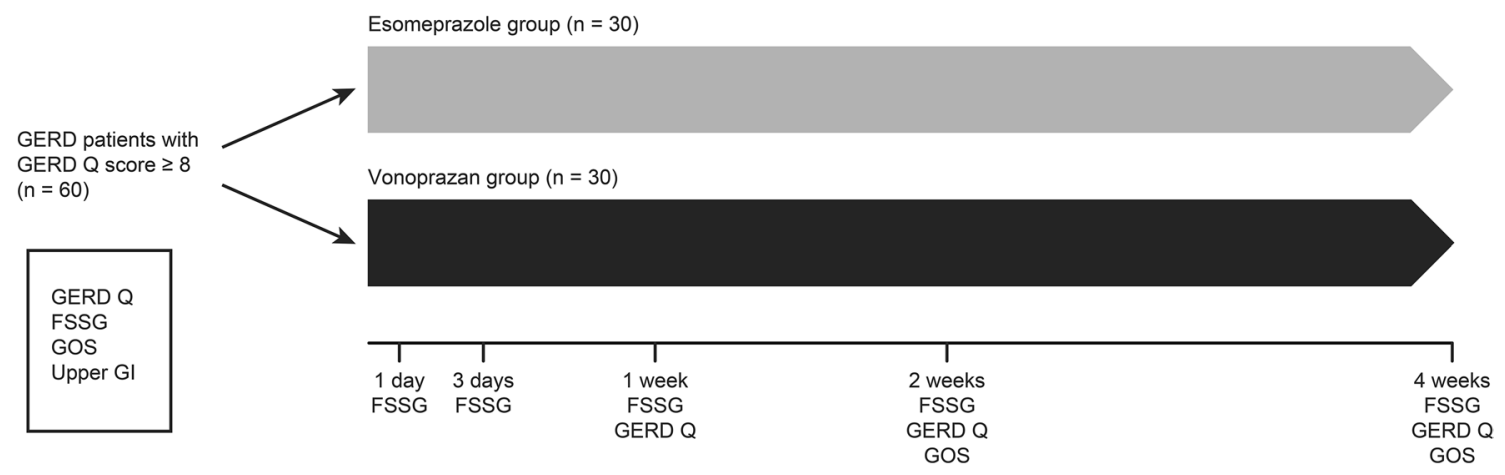

Fig. 1 Overview of the study design. GerdQ Gastroesophageal Reflux Disease Questionnaire; FSSG Frequency Scale for the Symptoms of GERD; GOS Global Overall Symptoms; Upper GI upper gastrointestinal endoscopy 
was verified that the exclusions did not change the primary results.

Table 1 shows patients' demographic and clinical characteristics. Body mass index (BMI) was significantly higher in the vonoprazan group than in the esomeprazole group. The other factors (mean age, sex, smoking, alcohol use, atrophy, and LA grade) were not significantly different between groups.

No significant difference was observed between the groups for the total FSSG, GerdQ, and GOS scores prior to treatment.

\section{GerdQ Score}

Sufficient relief was observed in $88.0 \%$ (95\% confidence interval (CI) $68.8-97.5 \%$ ) of patients in the esomeprazole group and $81.8 \%$ (95\% CI 59.7-94.8\%) of patients in the vonoprazan group after 4 weeks. Figure 2 shows the rates of complete resolution and sufficient relief at 1,2 , and 4 weeks. No significant difference was found between the two treatment groups.

We performed a stratified analysis of patients with NERD and erosive esophagitis. The proportions of patients with sufficient relief in NERD were 16.7, 66.7, and $66.7 \%$ of 13 patients in the esomeprazole group and 25.0, 75.0, and $75.0 \%$ of 9 patients in the vonoprazan group at 1,2 , and 4 weeks, respectively. The proportions of patients with complete resolution in NERD were $0,33.3$, and $66.7 \%$ in the esomeprazole group and $0,25.0$, and $50.0 \%$ in the vonoprazan group at 1,2, and 4 weeks, respectively. There were no significant differences in the rates of sufficient relief and complete resolution in NERD at 1, 2, or 4 weeks between the esomeprazole group and the vonoprazan group.

The proportions of patients with sufficient relief in erosive esophagitis were $33.3,100.0$, and $100.0 \%$ of 12 patients in the esomeprazole group and 50.0, 50.0, and $50.0 \%$ of 13 patients in the vonoprazan group at 1,2, and 4 weeks, respectively. The proportions of patients with complete resolution in erosive esophagitis were $0,33.3$, and $33.3 \%$ in the esomeprazole group and 50.0, 25.0, and $25.0 \%$ in the

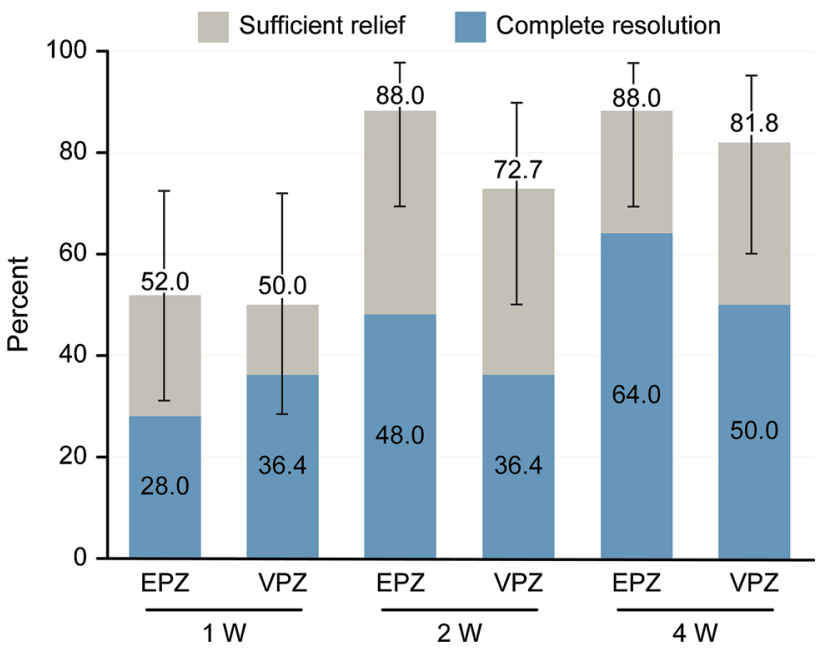

Fig. 2 Percentage of patients with complete resolution and sufficient relief as evaluated by the GerdQ after treatment with $20 \mathrm{mg}$ of esomeprazole once daily $(n=25)$ or $20 \mathrm{mg}$ of vonoprazan once daily $(n=22)$. Sufficient relief was defined by a score $\leq 1$ for each positive predictor question $(1,2,5$, and 6) on the GerdQ. Complete resolution was defined by a score of 0 for all questions. Error bars indicate $95 \%$ confidence intervals

vonoprazan group at 1,2, and 4 weeks, respectively. There were no significant differences in the rates of sufficient relief and complete resolution at 1,2 , or 4 weeks between the esomeprazole group and the vonoprazan group in erosive esophagitis.

\section{Changes in FSSG Scores}

Figure $3 \mathrm{a}, \mathrm{b}$ shows the changes in the total score, acidrelated symptom score, and dysmotility symptom score before and after treatment.

The mean FSSG total score, acid reflux-related symptom score, and dysmotility symptom score decreased from the 1st day after treatment initiation in both groups. However, changes between days 14 and 28 were small for all scores. No significant differences were observed between treatment groups.
Table 1 Patients' demographic data and severity of gastric atrophy and reflux esophagitis

\begin{tabular}{llll}
\hline & $\begin{array}{l}\text { Esomeprazole group } \\
(n=25)\end{array}$ & $\begin{array}{l}\text { Vonoprazan group } \\
(n=22)\end{array}$ & $P$ value \\
\hline Sex (male/female) & $10 / 15$ & $6 / 16$ & N.S. \\
Age (mean \pm SD, years) & $54.7 \pm 13.2$ & $58.0 \pm 13.8$ & N.S. \\
Body mass index (mean \pm SD) & $23.1 \pm 2.7$ & $25.3 \pm 4.0$ & 0.04 \\
Smoking (number, yes/no) & $10 / 15$ & $5 / 17$ & N.S. \\
Alcohol drinking (number, yes/no) & $14 / 11$ & $11 / 11$ & N.S. \\
Gastric atrophy (number, closed type/open type) & $19 / 6$ & $15 / 7$ & N.S. \\
LA classification grade (N or M/A/B/C/D) & $13 / 6 / 3 / 2 / 1$ & $9 / 7 / 3 / 3 / 0$ & N.S. \\
$\%$ of LA grade C or D & $12.0 \%$ & $10.5 \%$ & N.S. \\
\hline
\end{tabular}




\section{GOS Scores After Treatment}

Figure 4 shows the symptom resolution and relief rate of heartburn and regurgitation on the GOS scale, respectively. Sufficient relief was reported by $87.0 \%$ to $90.9 \%$ of patients in the esomeprazole group and $77.8 \%$ to $95.0 \%$ of patients in the vonoprazan group at 2 weeks, which demonstrates that most patients experienced improvement in the impact of GERD symptoms on daily activities within 2 weeks.

\section{Factors Associated with Symptom Relief}

The logistic regression analysis presented that the therapeutic efficacies of esomeprazole and vonoprazan were not affected by the degree of atrophy (closed type or open type), BMI, severe esophagitis, age, sex, drinking or smoking habits (data not shown).
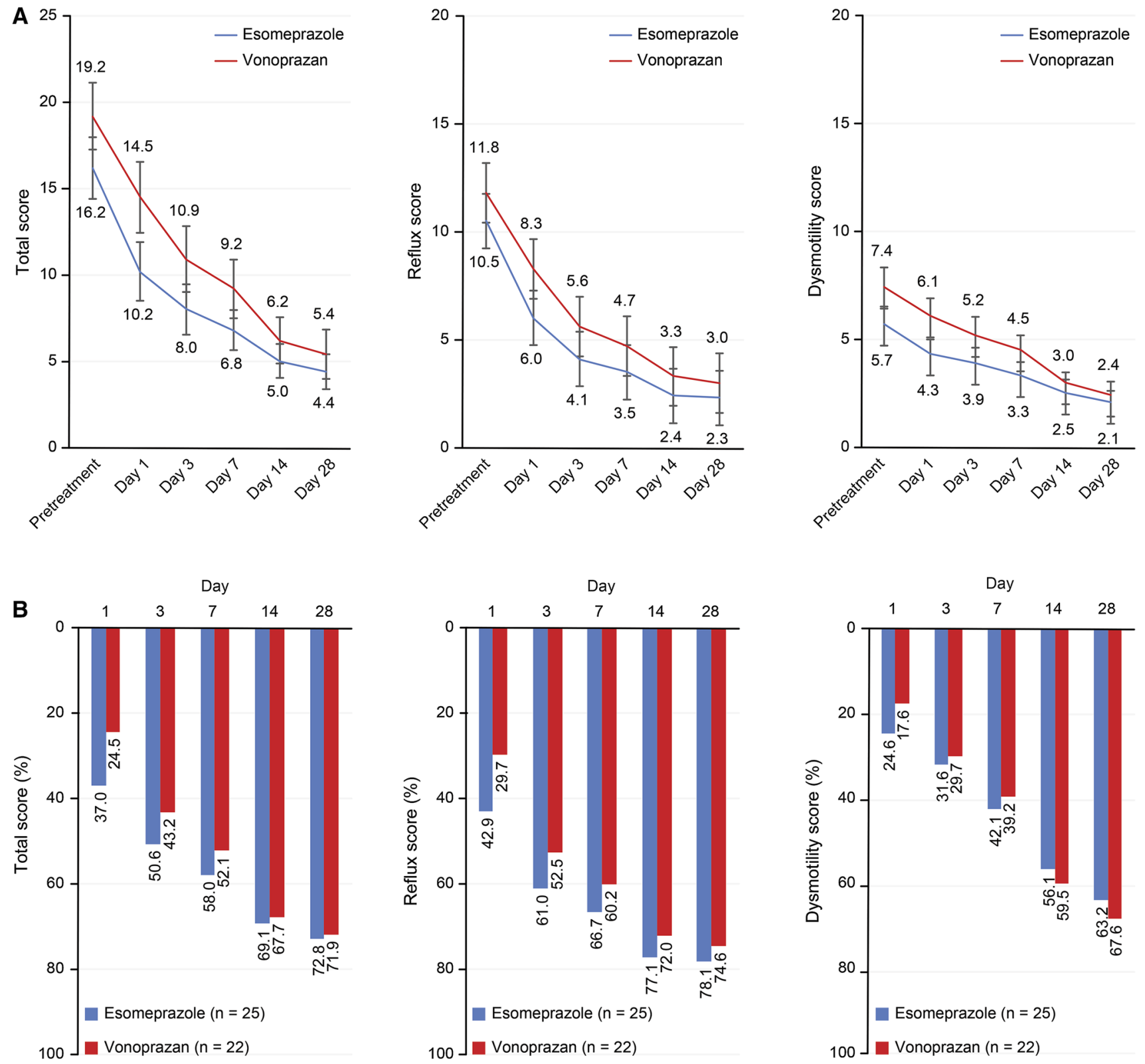

Fig. 3 a Changes in FSSG scores prior to and after treatment with $20 \mathrm{mg}$ of esomeprazole once daily $(n=25)$ or $20 \mathrm{mg}$ of vonoprazan once daily $(n=22)$. Each score is presented as the mean value and standard error. Reflux and dysmotility scores were defined according to Kusano et al. [11]. b Rate of decreased FSSG scores after treatment with $20 \mathrm{mg}$ of esomeprazole once daily $(n=25)$ or $20 \mathrm{mg}$ of vonoprazan once daily $(n=22)$. Reflux and dysmotility scores were defined according to Kusano et al. [11] 


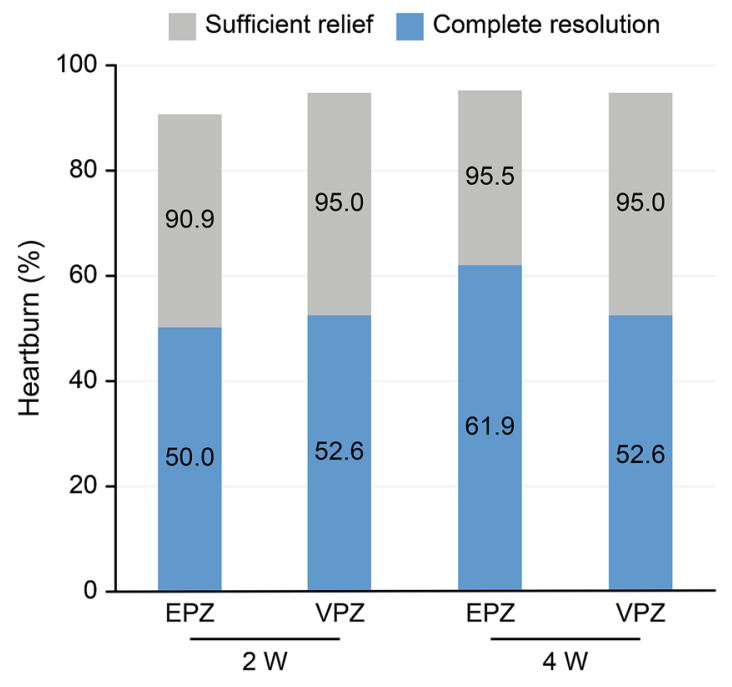

Fig. 4 Percentage of patients with complete resolution and sufficient relief as evaluated using the GOS scale after treatment with $20 \mathrm{mg}$ of esomeprazole once daily $(n=25)$ or $20 \mathrm{mg}$ of vonoprazan once daily

\section{Safety}

No AEs were reported during the study.

\section{Discussion}

In this study, we investigated the therapeutic effect of esomeprazole and vonoprazan as first-line treatments for early GERD symptom relief. Gastric acid inhibition is the most effective measure for GERD treatment. PPIs exhibit superior effects on GERD symptoms and are often selected as first-line treatments. Vonoprazan was introduced recently in Japan as the first authorized P-CAB agent, and it is expected to exhibit more pronounced efficacy in acid-related diseases.

Sakurai et al. [23] reported that the acid inhibition of vonoprazan was greater than that of esomeprazole and rabeprazole. The effect manifests quickly, and most patients achieve a gastric $\mathrm{pH}$ of at least 5 from the first day of treatment. The cure rate compared to conventional PPIs is particularly favorable in patients with severe reflux esophagitis [15].

In our study, esomeprazole and vonoprazan groups exhibited similar GERD symptom relief in all measurements.

Heartburn symptoms in GERD occur via two mechanisms. One of them is the "acid penetration theory" [24, 25]. Gastric acid, bile acid, and pepsin injure the esophageal mucosa and tight junction proteins in the esophageal epithelium. These factors penetrate the deep layers of the esophagus and directly activate nociceptors. The other mechanism is immunological. Acid and other factors, such as stress or sleep disturbance, contribute to esophageal mucosa

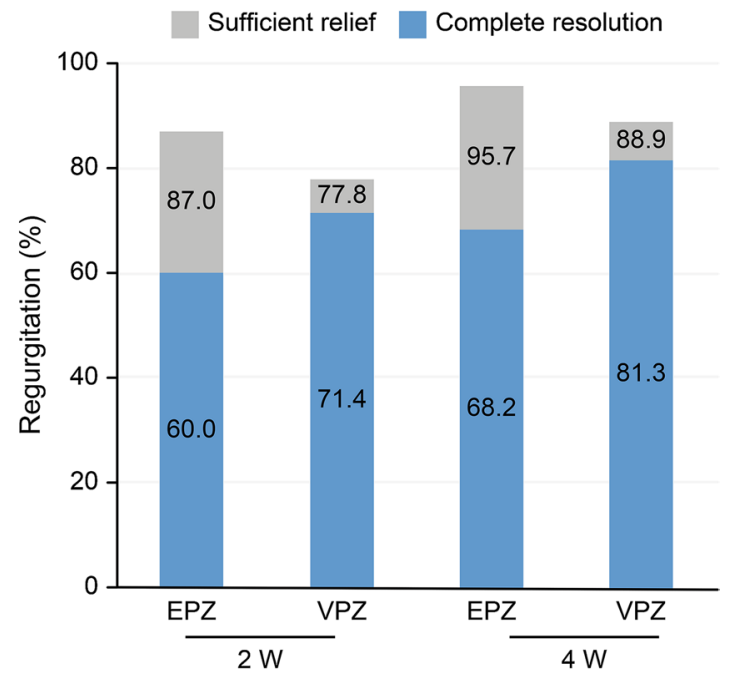

$(n=22)$. Sufficient relief was defined by a score $\leq 2$ for each heartburn or regurgitation question on the GOS scale. Complete resolution was defined by a score of 1 on the respective GOS scale question

permeability disturbances [26]. Inflammatory cytokines, such as interleukin-8 (IL-8) and IL-1 $\beta$, are secreted from the mucosal epithelium of the esophagus and increase the excitability of sensory nerves to produce esophageal hypersensitivity [27, 28]. This esophageal hypersensitivity is considered the cause of heartburn in GERD patients.

Heartburn involves an interplay between acid exposure and esophageal hypersensitivity. Abnormal acid exposure causes the predominant symptom in erosive esophagitis, while symptoms of NERD are related to the combination of acid exposure and esophageal hypersensitivity. Symptoms of functional heartburn do not correspond with reflux symptoms or reflux into the esophageal area, and thus esophageal hypersensitivity causes the predominant symptom [29].

Fennerty et al. reported that the heartburn severity of GERD symptoms did not correlate with severe erosive esophagitis, which was present in $31 \%$ of patients with severe heartburn compared to $23 \%$ of patients with moderate heartburn and $22 \%$ of patients with no or mild heartburn. The degree of heartburn severity exhibited similar percentages regardless of the presence of severe erosive esophagitis [30].

In our study, heartburn symptom relief regardless of the presence of severe erosive esophagitis (grades C and D) was similar between the esomeprazole and vonoprazan groups. Relief of heartburn symptoms in NERD was also similar between the esomeprazole and vonoprazan groups.

In a previous study, the proportions of patients with healed gastric or duodenal ulcers were similar between vonoprazan and lansoprazole groups at week 2 or week 4 [31]. In another study, the effectiveness of vonoprazan in preventing ulcer recurrence in patients receiving NSAIDs 
was noninferior to lansoprazole [32]. Based on these results, we think that both drugs have a sufficient 24-h intragastric $\mathrm{pH}>3$ holding time ratio to heal peptic ulcers. On the other hand, higher acid suppression might be necessary for esophageal mucosal lesions. Ashida et al. [15] reported that the proportion of erosive esophagitis healing was higher for vonoprazan compared with lansoprazole at 2 weeks and that vonoprazan was effective in patients with more severe esophagitis (LA classification grade C/D). The healing rate was supported by the report that the 24-h intragastric $\mathrm{pH}>4$ holding time ratio of vonoprazan was significantly higher than that of esomeprazole and rabeprazole on days 1 and 7 [23]. However, GERD symptoms are not associated with the degree of mucosal damage. Regarding this discrepancy, we think that GERD symptoms are influenced by both acid exposure and other factors such as esophageal hypersensitivity.

BMI was significantly higher in the vonoprazan group than in the esomeprazole group prior to treatment. However, the GerdQ, FSSG, and GOS scores were not significantly different between groups prior to treatment for obese or nonobese patients. Changes in the respective scores between groups were not significant after treatment (data not shown). Therefore, obesity had little influence on GERD symptoms. No factors related to the appearance of symptoms were detected in the multivariable analysis.

In the FSSG score, not only reflux scores but also dysmotility scores decreased from day 1 in both the esomeprazole and vonoprazan groups. The dysmotility symptoms of GERD patients may be caused by gastric acid. However, $\mathrm{pH}$ or $\mathrm{pH}$ impedance monitoring must be performed to determine whether the causality of the symptom is derived from abnormal acid exposure, i.e., whether it is due to reflux hypersensitivity or functional heartburn.

The strength of this study was that esomeprazole and vonoprazan showed similar effects as first-line treatments for the relief of typical symptoms of GERD and a plateau effect of each drug was observed on day 14. Meanwhile, the clinical benefit of treatment with vonoprazan over PPIs could not be presented clearly with the endpoint of relief of GERD symptoms, though it was reported that vonoprazan inhibited faster than and more highly gastric acid than PPIs. One of the reasons might have been explained by difference of $H$. pylori infection which we did not investigate the status though there was no significant difference of atrophic gastritis observed by endoscopy. Care should be taken with the interpretation of the results due to the limited sample size, open-label condition, and not including a placebo group. Several factors other than acid reflux should also be considered in the clinical endpoints. A large-scale, comparative study is necessary to verify the clinical benefits of stronger acid suppression than PPIs in the management of GERD symptoms.
Most Japanese GERD patients have NERD or grade A/B esophagitis [1], and treatments in many patients are initiated by the primary care physician. The results of this study provide evidence for the real-world practice of giving priority to the relief of symptoms.

In conclusion, there were no substantial differences in the sufficiency of symptom relief between esomeprazole and vonoprazan at any time point in this short-term study and the relief of symptom of each treatment could be assessed in 14 days as maximum effect.

Funding None.

\section{Compliance with ethical standards}

Conflict of interest The authors declare that they have no conflicts of interest related to this manuscript.

Open Access This article is distributed under the terms of the Creative Commons Attribution-NonCommercial 4.0 International License (http://creativecommons.org/licenses/by-nc/4.0/), which permits any noncommercial use, distribution, and reproduction in any medium, provided you give appropriate credit to the original author(s) and the source, provide a link to the Creative Commons license, and indicate if changes were made.

\section{References}

1. Fujiwara Y, Arakawa T. Epidemiology and clinical characteristics of GERD in the Japanese population. $J$ Gastroenterol. 2009;44:518-534.

2. Altomare A, Guarino MP, Cocca S, Emerenziani S, Cicala M. Gastroesophageal reflux disease: update on inflammation and symptom perception. World J Gastroenterol. 2013;19:6523-6528.

3. Armstrong D. Endoscopic evaluation of gastro-esophageal reflux disease. Yale J Biol Med. 1999;72:93-100.

4. Hongo M, Kinoshita Y, Miwa H, Ashida K. The demographic characteristics and health-related quality of life in a large cohort of reflux esophagitis patients in Japan with reference to the effect of lansoprazole: the REQUEST study. J Gastroenterol. 2008;43:920-927.

5. Pace F, Negrini C, Wiklund I, Rossi C, Savarino V, The Italian One Investigators Study Group. Quality of life in acute and maintenance treatment of non-erosive and mild erosive gastro-oesophageal reflux disease. Aliment Pharmacol Ther. 2005;22:349-356.

6. Iwakiri K, Kinoshita Y, Habu Y, et al. Evidence-based clinical practice guidelines for gastroesophageal reflux disease 2015. $J$ Gastroenterol. 2016;51:751-767.

7. Miner P Jr, Katz PO, Chen Y, Sostek M. Gastric acid control with esomeprazole, lansoprazole, omeprazole, pantoprazole, and rabeprazole: a five-way crossover study. Am J Gastroenterol. 2003;98:2616-2620.

8. Fass R, Shapiro M, Dekel R, Sewell J. Systematic review: proton-pump inhibitor failure in gastro-oesophageal reflux diseasewhere next? Aliment Pharmacol Ther. 2005;22:79-94.

9. Furuta T, Sugimoto M, Shirai N. Individualized therapy for gastroesophageal reflux disease: potential impact of pharmacogenetic testing based on CYP2C19. Mol Diagn Ther. 2012;16:223-234. 
10. Andersson K, Carlsson E. Potassium-competitive acid blockade: a new therapeutic strategy in acid-related diseases. Pharmacol Ther. 2005;108:294-307.

11. Drug approval review for vonoprazan fumarate (in Japanese). The Pharmaceuticals and Medical Devices Agency of Japan. 2014. Available at: http://www.pmda.go.jp/drugs/2014/P201400173/ index.html. Accessed May 28, 2015.

12. The Interview Form for Vonoprazan Fumarate (Takecab Tablets $10 \mathrm{mg}$ and $20 \mathrm{mg}$ ) ver. 3, (in Japanese). Takeda Pharmaceutical Company Limited. 2015. Available at: http://www.info. pmda.go.jp/go/interview/1/400256_2329030F1020_1_005._1F. Accessed May 28, 2015.

13. Prescribing information of Takecab tablets $10 \mathrm{mg}$ and $20 \mathrm{mg}$ ver. 2 (in Japanese). 2015. Available at: http://www.pmda.go.jp/PmdaS earch/iyakuDetail/ResultDataSet. Accessed May 28, 2015.

14. Jenkins H, Sakurai Y, Nishimura A, et al. Randomised clinical trial: safety, tolerability, Pharmacokinetics and pharmacodynamics of repeated doses of TAK-438 (vonoprazan), a novel potassium-competitive acid blocker, in healthy male subjects. Aliment Pharmacol Ther. 2015;41:636-648.

15. Ashida K, Sakurai Y, Hori T, et al. Randomised clinical trial: vonoprazan, a novel potassium-competitive acid blocker, vs. lansoprazole for the healing of erosive oesophagitis. Aliment Pharmacol Ther. 2016;43:240-251.

16. Jones R, Junghard O, Dent J, et al. Development of the Gerd $\mathrm{Q}$, a tool for the diagnosis and management of gastro-oesophageal reflux disease in primary care. Aliment Pharmacol Ther. 2009;15:1030-1038.

17. Suzuki H, Matsuzaki J, Okada S, Hirata K, Fukuhara S, Hibi T. Validation of the GerdQ questionnaire for the management of gastro-oesophageal reflux disease in Japan. U Eur Gastroenterol J. 2013;1:175-183.

18. Kusano M, Shimoyama Y, Sugimoto S, et al. Development and evaluation of FSSG: frequency scale for the symptoms of GERD. J Gastroenterol. 2004;39:888-891.

19. Veldhuyzen Van Zanten SJ, Chiba N, Armstrong D, et al. Validation of a 7-point global overall symptom scale to measure the severity of dyspepsia symptoms in clinical trials. Aliment Pharmacol Ther. 2006;23:521-529.

20. Lundell LR, Dent J, Bennett JR, et al. Endoscopic assessment of oesophagitis: clinical and functional correlates and further validation of the Los Angeles classification. Gut. 1999;45:172-180.

21. Miwa H, Yokoyama T, Hori K, et al. Interobserver agreement in endoscopic evaluation of reflux esophagitis using a modified Los
Angeles classification incorporating grades $\mathrm{N}$ and $\mathrm{M}$ : a validation study in a cohort of Japanese endoscopists. Dis Esophagus. 2008;21:355-363.

22. Kimura K, Takemoto T. An endoscopic recognition of the atrophic border and its significance in chronic gastritis. Endoscopy. 1969;1:87-97.

23. Sakurai Y, Mori Y, Okamoto H, et al. Acid-inhibitory effects of vonoprazan $20 \mathrm{mg}$ compared with esomeprazole $20 \mathrm{mg}$ or rabeprazole $10 \mathrm{mg}$ in healthy adult male subjects - a randomised open-label cross-over study. Aliment Pharmacol Ther. 2015;42:719-730.

24. Tobey NA, Carson JL, Alkiek RA, Orlando RC. Dilated intercellular spaces: a morphological feature of acid reflux-damaged human esophageal epithelium. Gastroenterology. 1996;111:1200-1205.

25. Barlow WJ, Orlando RC. The pathogenesis of heartburn in nonerosive reflux disease: a unifying hypothesis. Gastroenterology. 2005; 128:771-778.

26. Fass R, Naliboff BD, Fass SS, et al. The effect of auditory stress on perception of intraesophageal acid in patients with gastroesophageal reflux disease. Gastroenterology. 2008;134:696-705.

27. Souza RF, Huo X, Mittal V, et al. Gastroesophageal reflux might cause esophagitis through a cytokine-mediated mechanism rather than caustic acid injury. Gastroenterology. 2009;137:1776-1784.

28. Shan J, Oshima T, Fukui H, Watari J, Miwa H. Acidic deoxycholic acid and chenodeoxycholic acid induce interleukin-8 production through $\mathrm{p} 38$ mitogen-activated protein kinase and protein kinase A in a squamous epithelial model. $J$ Gastroenterol Hepatol. 2013;28:823-828.

29. Aziz Q, Fass R, Gyawali CP, Miwa H, Pandolfino JE, Zerbib F. Functional Esophageal disorders. Gastroenterology. 2016;150:1368-1379.

30. Fennerty MB, Johnson DA. Heartburn severity does not predict disease severity in patients with erosive esophagitis. Med. Gen Med. 2006;8:6.

31. Miwa H, Uedo N, Watari J, et al. Randomised clinical trial: efficacy and safety of vonoprazan vs. lansoprazole in patients with gastric or duodenal ulcers - results from two phase 3, non-inferiority randomised controlled trials. Aliment Pharmacol Ther. 2017;45:240-252.

32. Mizokami Y, Oda K, Funao N, et al. Vonoprazan prevents ulcer recurrence during long-term NSAID therapy: randomised, lansoprazole-controlled non-inferiority and single-blind extension study. Gut. 2018;67:1042-1051. 